\title{
Prognostic Factors and the Role of Adjuvant Chemotherapy in Post-curative Surgery for Dukes B and C Colon Cancers and Survival Outcomes: a Malaysian Experience
}

\author{
Astrid Sinarti Hassan ${ }^{1 *}$, Manimalar Naicker ${ }^{2}$, Khairul Hazdi Yusof ${ }^{3}$, Wan \\ Zamaniah Wan Ishak ${ }^{1}$
}

\begin{abstract}
Background: Adjuvant chemotherapy improves survival in Dukes C colon cancers post-curative resection. However, the evidence for a role with Dukes B lesions remains unproven despite frequent use for disease characterized by poor prognostic features. In view of limited Asia-specific data, this study aimed to determine survival outcomes and identify prognostic factors in a tertiary teaching hospital in Malaysia. Materials and Methods: A total of 116 subjects who underwent curative surgery with and without adjuvant chemotherapy for Duke B and C primary colon adenocarcinomas diagnosed from 2004-2009 were recruited and data were collected retrospectively. Five-year overall survival (OS) and disease free survival (DFS) were analysed using Kaplan-Meier survival analysis and log-rank (Mantel-Cox) test. Prognostic factors were determined using Cox proportional hazards regression with both univariate and multivariate analyses. $\underline{\text { Results: }}$ The survival analysis demonstrated a 5-year OS of $\mathbf{7 4 . 0 \%}$ for all patients, with $\mathbf{7 4 . 9 \%}$ for Dukes $\mathrm{C}$ subjects receiving chemotherapy compared to $28.6 \%$ in those not receiving chemotherapy $(p=0.001)$. For Dukes B disease, the 5-year survival rate was $82.6 \%$ compared to $\mathbf{7 5 . 0 \%}$ for subjects receiving and not receiving chemotherapy, respectively $(\mathrm{p}=\mathbf{0 . 1 7})$. Independent prognostic factors identified included a CEA level more than $3.5 \mathrm{ng} / \mathrm{ml}$ (hazard ratio $(\mathrm{HR})=4.78 ; \mathrm{p}=0.008)$, serosal involvement $(H R=3.75 ; p=0.028)$ and completion of chemotherapy $(H R=0.20 ; p=0.007)$. Conclusions: In a regional context, this study supports current evidence from the West that adjuvant chemotherapy improves survival in Dukes $\mathrm{C}$ colon cancers post curative surgery. However, although a clear benefit has yet to be proven for Dukes B disease, our results suggest survival improvement in selected cases.
\end{abstract}

Keywords: Colon cancer - Dukes B - Dukes C - adjuvant chemotherapy

Asian Pac J Cancer Prev, 16 (6), 2237-2243

\section{Introduction}

Colorectal cancer is the second commonest cancer after breast cancer. It is also the second most common cancer in males and females in Malaysia. In Malaysia, a total of 2,246 colorectal cases were diagnosed in 2007 and represent $12.3 \%$ of all cases reported. The incidence of colorectal cancer increases with age and slightly higher among males compared to females and it is highest among Chinese (Ariffin and Nor Saleha, 2011).

Treatment for colon cancer is currently dictated by stage. Curative surgery with resection of macroscopic and microscopic evidence of the tumour in addition to en bloc removal of mesenteric lymph nodes is the primary treatment for loco-regional disease and depending on the extent of disease there may be a role for adjuvant therapy. Dukes A (stage I) patients are treated with surgery alone while patients with Dukes C (stage III) have improved survival with adjuvant chemotherapy. The role of adjuvant treatment in the management of Dukes B (T3 or T4 N0 (stage II)) patients is, however, controversial because of conflicting results from clinical trials and population based studies(Morris et al., 2007). The Multicenter International Study of Oxaliplatin/5-Fluorouracil/Leucovorin in the Adjuvant Treatment of Colon Cancer (MOSAIC) trial demonstrated the use of 5-Fluorouracil plus Leucovorin in adjuvant setting reduces the mortality rate by 33 percent among patients with Dukes C (stage III) colon cancer (Andre et al., 2004). In the MOSAIC trial, the significant disease free survival (DFS) and overall survival (OS) benefits observed with the addition of Oxaliplatin to 5-Fluorouracil/Leucovorin in the overall population were driven entirely by the favorable effect in the subgroup of patients with stage III disease. For stage II patients, there was no statistically significant improvement in 5-year DFS and 6-year OS (Andre et al., 2009).

The decision to administer adjuvant chemotherapy are based upon the intra- and postoperative Dukes stages in 
which Dukes B and Dukes C disease imply the absence of lymph nodes disease or the presence of tumour in the lymph nodes, respectively (Benson et al., 2004). Subjects with Dukes $C$ are offered adjuvant chemotherapy; usually utilising 5-Fluorouracil-based treatment (O'Connor et al., 2011).

For Dukes B colon cancer without adverse prognostic features chemotherapy is not offered as similar benefits has not been convincingly demonstrated (Quasar Collaborative et al., 2007; Quah et al., 2008; O'Connor et al., 2011). However, in high risk Dukes B disease, the administration of adjuvant chemotherapy is not uncommon despite uncertain impact on survival (Schrag et al., 2002; Wilkinson et al., 2010). The American Society of Clinical Oncology guidelines suggest that certain poor prognostic features such as elevated preoperative carcinoembryonic antigen (CEA), presence of bowel obstruction or perforation on presentation, need for an emergency operation, inadequate nodal evaluation ( $<12$ nodes), perineural and/or lymphovascular invasion and tumour differentiation might reasonably prompt practitioners to consider therapy (Benson et al., 2004; Quah et al., 2008). In particular, (Galizia et al., 2009) has quoted a preoperative carcinoembryonic antigen (CEA) > $3.5 \mathrm{ng} / \mathrm{ml}$ as the cut-off point as a poor prognostic feature. In the MOSAIC trial, the multivariate analysis for stage II and stage III prognostic factors remained statistically significant for age, lymph node involvement, T stage, tumor obstruction, and differentiation (Andre et al., 2009).

In light of deficient Asia-specific data, this study aims to determine the survival outcomes in subjects with Dukes $\mathrm{B}$ and $\mathrm{C}$ subjects who underwent curative resection with and without adjuvant chemotherapy in a teaching hospital in Malaysia over a period of 6 years from January 2004 to December 2009.

\section{Materials and Methods}

\section{Patient and tumour characteristics}

All subjects undergoing surgical resection for a primary colon adenocarcinoma with Dukes B and Dukes C diseases were entered retrospectively into a database from a single, large teaching hospital in the Peninsular Malaysia (University Malaya Medical Centre UMMC). The study has received prior approval by the Medical Ethics Committee UMMC (Ref: 1010.40). Only subjects with pathological studies confirmed colon adenocarcinoma were included whilst subjects with a history of current and/or previous other malignant disorders were excluded. Data collected included patients' demographic, CEA levels at presentation, macroscopic surgical features, histopathology (such as tumour stage, number of lymph nodes harvested) and use of adjuvant chemotherapy.

The decision to administer adjuvant chemotherapy was determined by a multidisplinary team in which factors such as final pathology, established prognostic factors, clinical condition and anticipated prognosis were reviewed. The standard chemotherapy regime was based on 5-Fluorouracil/Leucovorin alone or in combination with Irinotecan or Oxaliplatin, or Oxaliplatin/Capecitabine alone or Capecitabine alone. Follow-up data were retrieved from patient charts including disease recurrence based on the RECIST criteria (Response Evaluation Criteria in Solid Tumours), colonoscopy results and subsequent confirmation histologically. Date and cause of death were retrieved from the National Registry Department.

\section{Statistical analysis}

Survival from the date of operation was calculated using the Kaplan-Meier survival curves, and the log rank (Mantel-Cox) test to compare survival between each curve for both overall survival (OS) and disease-free survival (DFS). OS was defined from the time of surgery to the time of death from any cause whilst DFS was defined from the time of surgery to the time of development of first evidence of local recurrence or distant metastasis. For OS, subjects are censored if they were still alive at the last follow-up. 5-year survival rate were calculated using the Kaplan Meier survival curves.

Chi-square test was used to compare differences in the patient characteristics between groups. Univariate and multivariate analysis using the Cox regression model was performed on each categorical variable to identify factors influencing overall survival as the end point. The SPSS Version 20 was used for all the statistical analysis in which the significance level was specified as $\mathrm{p}<0.05$ with $95 \%$ confidence interval (CI) for two-tailed analyses.

\section{Results}

Between 2004-2009, a total of 116 subjects $(n=54$ females, 62 males) with Dukes $B$ and $C$ receiving curative resection were recruited for data analysis with a median age 64.3 years (range 17- 82 years). There were a higher percentage $(69 \%)$ of subjects who were ethnic Chinese $(n=80)$ in the study but this was comparable across the groups with different Dukes stage with or without adjuvant chemotherapy. Patients' characteristics are depicted in Table 1.

Forty-four and 72 subjects were diagnosed postoperatively as stage Dukes B and Dukes C diseases respectively. Within this cohort, there was a higher rate of emergency resection in subjects eventually receiving adjuvant chemotherapy. Whilst $80 \%$ of subjects with Duke $\mathrm{C}$ disease received chemotherapy, only two third of subjects with Dukes B disease received chemotherapy based on poor prognostic features as per current recommendation and predictive prognosis based on co-morbidity and overall health (Schrag et al., 2002; Wilkinson et al., 2010). There were no meaningful differences detected taking into account all confounding factors including diabetes mellitus; cardiac disease, asthma etc that may impact on the 5-year survival analysis.

Meanwhile in the group with Dukes B patients, 32\% patients with obstruction received chemotherapy. In Dukes B subjects not receiving chemotherapy, the tumour grades were exclusively either well- or moderately-differentiated. Eighty-nine percent of Dukes B and $74.1 \%$ of Dukes C subjects which, underwent inadequate $\mathrm{LN}$ evaluation $(<12)$ received chemotherapy, leading to an average mean number of total LN evaluated in between 10.1 to 11.3. No statistical differences were observed for the involvement 
of perineural and lymphovascular invasion between the different groups. No perioperative mortality was reported.

\section{Outcomes and survival analysis}

At median follow up of 84 months, the Kaplan-Meier analysis conducted demonstrated a 5-year overall survival (OS) rate (all-cause mortality) of $74.0 \%$ respectively for all patients. Meanwhile, the 5-year survival rate was $86 \%$ for Dukes B and $66.7 \%$ correspondingly for Dukes $\mathrm{C}(\mathrm{p}=0.001)$

In subjects who did not receive chemotherapy, the 5- year survival rate was $75.0 \%$ with Dukes B, and $28.6 \%$ with Dukes C ( $\mathrm{p}=0.001)$ (Table 2). In Dukes B subjects receiving chemotherapy, the 5-year survival rate was $82.6 \%(\mathrm{HR}=0.36$; 95\%CI, 0.08-1.64; $\mathrm{p}=0.188)$. No statistical differences were observed in Dukes B subjects receiving chemotherapy compared to their counterparts not receiving chemotherapy (Figure 1). The median survival for overall survival was not reached at the time this manuscript was written. The 5-year survival rate for
Dukes $C$ patients receiving chemotherapy was $74.9 \%$ $(\mathrm{HR}=0.23 ; 95 \% \mathrm{CI}, 0.109-0.50 ; \mathrm{p}=0.001)$. The median survival time of $18.0 \pm 14.9$ (95\% CI 00.0-47.3) months was reached for Dukes $\mathrm{C}$ patients without chemotherapy. The same significant $(\mathrm{p}=0.001)$ were also detected for disease free survival in those receiving chemotherapy compared to their counterparts.

Based on the American Society of Clinical Oncology guidelines, multiple prognostic factors were analysed. On univariate analysis, CEA levels more than $3.5 \mathrm{ng} /$ $\mathrm{ml}$ (HR 4.83, $\mathrm{p}=0.005$ ), inadequate LN evaluated (less than 12) (HR 2.78, p=0 .09) and serosal involvement (HR $2.39, \mathrm{p}=0.09$ ) were significant factors for overall survival (Table 3). Meanwhile, completion of chemotherapy is associated with a good prognostic significance (HR 0.09, $\mathrm{p}=0.005$ ) for both Dukes B and C. These prognostic factors were similarly replicated on the multivariate regression analysis for CEA levels more than $3.5 \mathrm{ng} / \mathrm{ml}$ (HR 4.78, $\mathrm{p}=0.008)$ and serosal involvement (HR 3.75, $\mathrm{p}=0.028)$ albeit inadequate LN examined (HR 2.32, $\mathrm{p}=0.15$ ),

Table 1. Baseline Demographic and Tumour Characteristics of Individuals which Underwent Curative Resection with Duke B and C, in Subgroups with and without Adjuvant Chemotherapy

\begin{tabular}{|c|c|c|c|c|c|}
\hline & & \multicolumn{2}{|c|}{ DUKE B n=44 } & \multicolumn{2}{|c|}{ DUKE C n=72 } \\
\hline & & $\begin{array}{c}\text { No Chemo } \\
n=16\end{array}$ & $\begin{array}{c}\text { Chemo } \\
n=28\end{array}$ & $\begin{array}{c}\text { No Chemo } \\
n=14\end{array}$ & $\begin{array}{c}\text { Chemo } \\
n=58\end{array}$ \\
\hline Age, mean (SD) & & $68.7(9.3)$ & $56.1(16.9)$ & $53.6(19.3)$ & $60.7(14.0)$ \\
\hline Male sex, n (\%) & & $9(56)$ & $14(50)$ & $8(57)$ & $31(53)$ \\
\hline Female sex, n (\%) & & $7(44)$ & $14(50)$ & $6(43)$ & $27(47)$ \\
\hline \multirow[t]{4}{*}{ Race, n (\%) } & Malay & $2(12)$ & $6(21)$ & $5(36)$ & $9(16)$ \\
\hline & Chinese & $12(76)$ & $19(68)$ & $6(43)$ & $43(74)$ \\
\hline & Indian & $2(12)$ & $2(7)$ & $1(7)$ & $5(8)$ \\
\hline & Others & $0(0)$ & $1(4)$ & $2(14)$ & $1(2)$ \\
\hline \multirow[t]{9}{*}{ Co-morbidity, n (\%) } & Total & $11(69)$ & $9(32)$ & $3(21)$ & $29(50)$ \\
\hline & DM & 3 (19) & $5(18)$ & $2(14)$ & $12(21)$ \\
\hline & Hypertension & $8(50)$ & $7(25)$ & $3(21)$ & $18(31)$ \\
\hline & Hyperlipidaemia & $2(12)$ & $0(0)$ & $0(0)$ & $2(3)$ \\
\hline & IHD & $3(19)$ & $1(4)$ & $0(0)$ & $4(7)$ \\
\hline & CVA & $1(6)$ & $0(0)$ & $0(0)$ & $1(2)$ \\
\hline & Asthma & $0(0)$ & $1(4)$ & $0(0)$ & $3(5)$ \\
\hline & Gout & $1(6)$ & $0(0)$ & $0(0)$ & $1(2)$ \\
\hline & $\mathrm{BPH}$ & $1(6)$ & $0(0)$ & $0(0)$ & $1(2)$ \\
\hline \multirow[t]{3}{*}{ Tumour characteristics, n (\%) } & CEA, mean (SD)* & $3.23(2.3)$ & $2.82(2.0)$ & $32.9(69.1)$ & $6.08(16.5)$ \\
\hline & Obstruction & $2(12)$ & $9(32)$ & $1(7)$ & $18(31)$ \\
\hline & Perforation & $1(6)$ & $3(11)$ & $1(7)$ & $5(8)$ \\
\hline \multirow[t]{3}{*}{ Tumour grade differentiation } & Well & $5(31)$ & $3(11)$ & $3(21)$ & $4(7)$ \\
\hline & Moderate & $11(69)$ & $21(75)$ & $10(71)$ & $52(90)$ \\
\hline & Poor & $0(0)$ & $4(14)$ & $1(7)$ & $2(3)$ \\
\hline LN evaluated, mean (SD)* & & $11.2(7.7)$ & $10.1(4.8)$ & $10.3(6.1)$ & $11.3(6.12)$ \\
\hline Serosal involvement & & $10(63)$ & $12(43)$ & $10(71)$ & $34(59)$ \\
\hline LVI involvement & & $1(6)$ & $5(18)$ & $7(50)$ & $22(38)$ \\
\hline Perineural invasion & & $1(6)$ & $2(7)$ & $3(21)$ & $5(9)$ \\
\hline Emergency surgery & & $2(12)$ & $9(32)$ & $2(14)$ & $19(33)$ \\
\hline
\end{tabular}

*Percentages may not sum up to $100 \%$ due to rounding. Abbreviations: Chemo, chemotherapy; SD, standard deviation; DM, diabetes mellitus; IHD, ischaemic heart disease; CVA, cerebrovascular accident; BPH, benign prostatic hypertrophy; CEA, carcinoembryonic antigen; LN, lymph node; LVI, lymphovascular invasion

Table 2. Survival Analysis in Patients with Duke B and C Undergoing Curative Surgery with and without Adjuvant Chemotherapy

\begin{tabular}{llccc}
\hline & & No chemotherapy & Chemotherapy & Hazard ratio \\
\hline Dukes B & 5-year survival (\%) & 75 & 82.6 & 0.36 \\
& Confidence Interval & $75.0-125.4$ & $110.2-127.6$ & $0.08-1.64$ \\
\multirow{2}{*}{ Dukes C } & 5-year survival (\%) & 28.6 & 74.9 & 0.23 \\
& Confidence Interval & $23.1-61.4$ & $76.2-98.2$ & $0.11-0.50$ \\
\hline
\end{tabular}


Table 3. Univariate and Multivariate Analysis of Prognostic Factors for Overall Survival using the Cox Regression Method

\begin{tabular}{|c|c|c|c|c|c|c|c|}
\hline \multirow[t]{2}{*}{$\overline{\text { Variabl }}$} & & \multicolumn{3}{|c|}{ Univariate } & \multicolumn{3}{|c|}{ Multivariate } \\
\hline & & HR & $95 \% \mathrm{CI}$ & p-value & HR & $95 \% \mathrm{CI}$ & p-value \\
\hline \multirow[t]{13}{*}{ Age } & & 0.99 & $0.50-1.94$ & 0.97 & & & \\
\hline & Group $1 ;<30$ & N/A & & & 0.76 & $0.06-8.72$ & 0.83 \\
\hline & Group $2 ; 31-50$ & N/A & & & 0.42 & $0.07-2.75$ & 0.37 \\
\hline & Group 3; 51-80 & N/A & & & 1.24 & $0.38-4.06$ & 0.72 \\
\hline & Group $4 ;>70$ & N/A & & & 1.45 & $0.46-4.78$ & 0.48 \\
\hline & Gender (M vs F) & 1.44 & $0.49-4.14$ & 0.5 & 0.61 & $0.19-1.88$ & 0.39 \\
\hline & CEA $>3.5$ & 4.83 & $1.62-14.39$ & 0.005 & 4.78 & $1.51-14.89$ & 0.008 \\
\hline & Obstruction & 2 & $0.76-5.31$ & 0.16 & 1.89 & $0.69-5.15$ & 0.22 \\
\hline & Perforation & 0.86 & $0.11-6.99$ & 0.88 & 0.59 & $0.07-5.10$ & 0.63 \\
\hline & LN Evaluated $<12$ & 2.78 & $0.83-9.30$ & 0.09 & 2.32 & $0.74-7.25$ & 0.15 \\
\hline & Serosal involvement & 2.39 & $0.85-6.71$ & 0.09 & 3.75 & $1.15-12.25$ & 0.028 \\
\hline & LVI & 0.89 & $0.30-2.61$ & 0.84 & 1.11 & $0.39-3.15$ & 0.84 \\
\hline & Chemo completion & 0.09 & $0.02-0.48$ & 0.005 & 0.2 & $0.06-0.64$ & 0.007 \\
\hline
\end{tabular}

Abbreviations: HR, hazard ratio; CI, confidence interval; N/A, not applicable; Chemo, chemotherapy; M vs F, male versus female; CEA, carcinoembryonic antigen; LN, lymph node; LVI, lymphovascular invasion
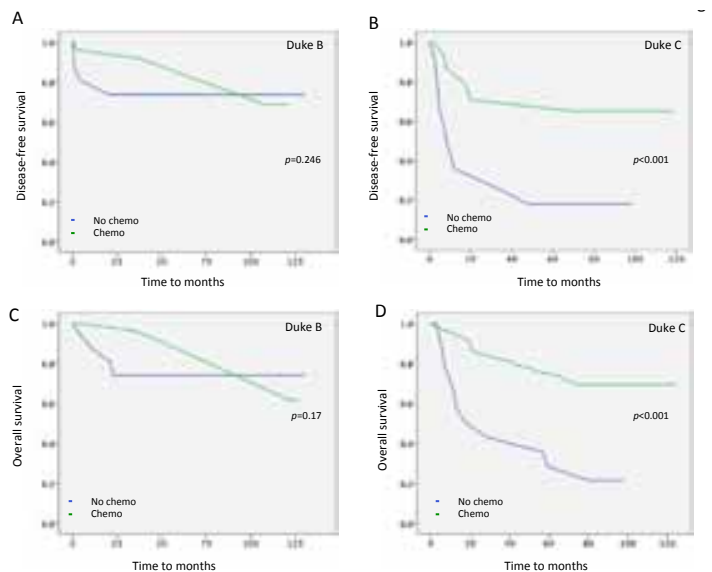

Figure 1.A,B, Disease-free and C,D, Overall Survival in Individuals with Dukes B and C Respectively, Receiving or not Receiving Adjuvant Chemotherapy

which was not significant. Completion of chemotherapy was again observed to be a good prognostic feature (HR $0.20, \mathrm{p}=0.007)$. There was a trend for increasing age as an adverse prognostic factor; however, this was not statistically significant.

\section{Discussion}

The incidence of colon cancer in South East Asia has assumed global trends, which is influenced by lifestyle and environmental factors such as smoking, obesity and physical inactivity (Chong et al., 2009; Tsukuma et al., 2011; Yahaya et al., 2011). However, Asia-specific data are lacking to reflect treatment outcomes based on evidence and recommendations from the West. Ethnicities and nationalities have also been proven as factor in the upward trend in global incidence and therefore, national and regional studies are necessary to improve local treatment outcomes (Magaji et al., 2014; Shah et al., 2014). The purpose of this study is to report the South East Asia and Malaysian experience especially in regards to mortality and survival outcomes in subjects who underwent curative resection for Dukes $\mathrm{B}$ and $\mathrm{C}$ with and without adjuvant chemotherapy based on the current recommendations.

Complete surgical resection is the primary treatment for patients with loco-regional colon cancer. The Quick and Simple and Reliable (QUASAR) prospective trial failed to demonstrate survival benefit for stage II colon cancer (HR 0.86; 95\%CI, 0.66 to 1.12) (Quasar Collaborative et al., 2007). A meta-analysis from five National Surgical Bowel and Breast Project trials reported a survival advantage of chemotherapy for this population (HR 0.58; 95\%CI, 0.48 to 0.71) (Wilkinson et al., 2010). Another study retrospectively reported 24,847 Medicare patients with Dukes B (stage II) with $20 \%$ receiving adjuvant chemotherapy, which revealed no substantial improvement in overall survival. Interestingly, this group reported that the use of chemotherapy does not differ between Dukes B (stage II) patients with or without poor prognostic features (O'Connor et al., 2011). A larger meta-analysis of 12 clinical trials, which resulted in the consensus recommendations from American Society of Clinical Oncology showed that existing trial data suggest $2-4 \%$ increase in absolute survival with no statistical significant improvement. Therefore this does not support routine use of chemotherapy in patients with stage II disease except for the presence of poor prognostic features as outlined (Benson et al., 2004).

Not an uncommon practice, adjuvant chemotherapy would be offered to subjects of Dukes B disease with poor prognostic features, which may reflect reverse causality for a poorer prognosis and affect survival analyses, with more meaningful statistical differences. In addition, any potential benefit may be obscured by other factors such as heterogeneity of the subgroups, insufficient number of patients in clinical trials, competing co-morbidities causing non-cancer related deaths and under-staging of subjects with inadequate nodal resection (Buyse and Piedbois, 2001). Due to inconsistency in evidence, physician's belief over the net benefit of adjuvant therapies for Dukes B cancer also remains divided (Wong et al., 2014).

For this study, several comments needed to be highlighted with the patient characteristics in this cohort. First, the median age is much younger than previously reported in which the median age of colon cancer diagnosis is 70 years. This may explain the relative high utilization 
of adjuvant chemotherapy particularly in patients with Dukes B disease. We also observed a higher incidence in Chinese patients for both Dukes B and Dukes C, in line with previous observations and reported by the Malaysian National Cancer Registry (Magaji et al., 2014). We propose two hypothesis; first in which ethnicity may play a role in the aetiology of colon cancer in Asia (Yee et al., 2009; Pourhoseingholi, 2012) and second, the possibility that the Chinese in our population are more likely to demonstrate health-related help-seeking behaviour and/or have better access to health care. The trend for increasing age as an adverse prognostic factor was not observed and may reflect the competing risk of co-morbidity coupled with previous reports suggesting that young subjects diagnosed with colon cancer have a worse survival (O'Connell et al., 2004; Elsamany et al., 2014).

We showed here that adjuvant chemotherapy improved overall survival by $46 \%$ for 5 -year analysis for Dukes C disease, supporting current guidelines. A smaller benefit of $8 \%$ was correspondingly observed for Dukes B disease. In the QUASAR study, there was a 5-year survival benefit of $3.6 \%$ in Dukes B (stage II), however it was limited by the inclusion of patients who had rectal cancer with relatively short median follow up (McKenzie et al., 2011). Albeit statistically significant difference detected, this observation demonstrates a better outcome than the 2-5\% improvement previously reported (Quasar Collaborative et al., 2007; O'Connor et al., 2011). In our study, only 44 patients with Dukes B were being analysed, in which 28 patients have received adjuvant chemotherapy and only 16 patients has not received adjuvant treatment. The 5 -year survival rate in these patients were $82.6 \%$ in the chemotherapy arm and $75 \%$ in the non chemotherapy arm, giving the absolute 5-year benefit of $7.6 \%$ in favour of adjuvant chemotherapy treatment. If we analysed the Kaplan-Meier survival curve for Dukes B (figure $1 \mathrm{~A}$ and C), the survival line of both arm actually crossed each other after 75 months, suggesting that adjuvant treatment with chemotherapy actually provided 5-year survival benefit for the patients. However in view of the small number of patients in this cohort, we need more data to support our theory.

It must be emphasised that this study only recruits subjects who underwent curative resection for Dukes $\mathrm{B}$ and $\mathrm{C}$ disease and therefore, the outcomes should not be generalised to subjects who did not receive surgical treatment. A previous epidemiologic study however has reported that the majority of our patients received surgery; where the aim was either curative or surgical palliation, reflecting a more centre-specific practice that may depend on local resources and staffs (Magaji et al., 2014).

The univariate and multivariate analyses supported previous studies, confirming the prognostic determinants of colon adenocarcinoma such as serosal involvement as an independent prognostic factor (Shepherd et al., 1997; Compton et al., 2000). A recent study concluded that T4N0 (Stage IIB/C) colon cancer had a paradoxically worse oncologic outcomes than T1-2N1 (Stage IIIA) disease regardless of adjuvant chemotherapy with 5-year OS of $87.1 \%$ vs $93.2 \%$ (Kim et al., 2015). Therefore, poor prognostic features in which the decision for adjuvant chemotherapy is based on, may cause a reverse causality and impact on the survival outcomes. Future studies are required to identify the efficacy of adjuvant chemotherapy for specific TNM staging, which may improve current practice. For example, another study reported that perineural invasion is the only prognostic factor affecting DFS and OS in patients with T3N0 colon cancer (Yun et al., 2014).

Higher levels of preoperative CEA levels have been highlighted as a poor prognostic indicator reflecting more locally advanced disease. Like other prognostic features outlined, it is unclear how they influence chemotherapy outcomes and survival benefit (Compton et al., 2000). Another independent prognostic factor identified in this study was the completion of chemotherapy. However, the question as whether the true efficacy of chemotherapy versus those who stop chemotherapy earlier may have other features related to poor survival (older age, side effects) remains unanswered. Variation of outcomes may also be explained by different regimen of chemotherapy that was utilized in this study as well. Although infusional 5-Fluorouracil/Leucovorin plus Oxaliplatin is an established chemotherapy regime for adjuvant colon cancer, Capecitabine/Oxaliplatin has been shown to be non-inferior and better adherence to treatment and well tolerated with better quality of life in East Asian patients (Chiu et al., 2014).

It has been previously demonstrated that lymph node assessment postoperatively represents a major prognostic factor (Rosenberg et al., 2008). The number of resected nodes has been demonstrated to be an independent prognostic factor related to long-term outcome (Vather et al., 2009). According to the guidelines from AJCC, a minimum of 12 lymph nodes must be retrieved and examined for accurate staging. Furthermore, The AJCC and The College of American Pathologists have recommended examination of a minimum of 12 lymph nodes to accurately identify stage II colorectal cancers. For stage II colon cancer, if initially, less than 12 lymph nodes were identified, it is recommended that the pathologist resubmit more tissue of potential lymph nodes from the said specimen. If 12 lymph nodes were still not identified, a comment in the report should indicate that an extensive search for lymph nodes was undertaken (Edge and Compton, 2010). The survival rates improve as the number harvested nodes increase (Chang et al., 2007). Previous studies have shown that adjuvant chemotherapy improves both disease-free and survival rates only in metastatic node colon cancers (positive LN, stage III). This lead to experts recommending adjuvant chemotherapy in node-negative colon cancers who had inadequate lymphadenectomy (Chen and Bilchik, 2006).

In our cohort, the average mean number of total LN evaluated is less than 12 , however cases of insufficiently retrieved LN are not infrequent among locally advanced colon cancer subjects which may suggest inadequate nodal evaluation. Recent studies have shown that the ratio of involved to the total resected LN is a better prognostic predictor than pathological node classification and may improve the clinical decision for adjuvant chemotherapy (Nadoshan et al., 2013). 
Other characteristics that have been proven to be associated with worse outcomes however have not been predictive of a successful response to adjuvant chemotherapy post curative resection, which highlights the need to identify other prognostic factors that may not necessarily rely on tumour-specific features. A recent study demonstrates a high association between tumour grading and recurrence which can be explained in this study where we observed in Dukes B subjects not receiving chemotherapy, the tumour grades were exclusively either well- or moderately-differentiated (Omranipour et al., 2014). We conclude that the poor prognostic features considered in this study and as outlined in the guidelines for the consideration of adjuvant chemotherapy represent an imprecise mechanism for not only identifying patients with high risk Dukes B cancer but also the true predictors for the outcome of adjuvant chemotherapy.

Other strengths of this study include data encompassing patients treated in a tertiary referral hospital with a functional multi-disciplinary team dedicated to the care of colon cancers and consistency of histopathology reporting. The limitation of this study includes the retrospective nature of data collection in a single institution with an average sample size of patients for each subgroup. In addition, recent epidemiologic studies have identified other modifiable risk factors such as physical inactivity, greater BMI and socioeconomic status, which were not explored (Kong et al., 2010; Morrison et al., 2013). A large prospective and randomised study for genetic factors may demonstrate a clearer benefit however require substantial resources and time. Recent evidence from a meta-analysis study suggests that genetic factor of 5,10- Methylenetrahydrofolate Reductase (MTHFR) polymorphisms may play a role in the protection for colon cancer (Fang et al., 2014). Meanwhile, a recent study suggested that detection of K-ras mutation subgroup in specific codons were significantly associated with early recurrence post curative surgery (Bozkurt et al., 2014).

In the context of a regional setting, this study is the first reporting mortality outcomes in subjects with Dukes B and Dukes $\mathrm{C}$ diseases receiving treatment based on current consensus. Importantly, this study confirms evidence and specific recommendations from the West that adjuvant chemotherapy improves survival in subjects with Dukes $\mathrm{C}$ disease receiving curative resection in South East Asia. There is also a trend that a smaller benefit is extended to Dukes B disease with poor prognostic factors however this is not statistically significant, adding to the body of evidence that adjuvant chemotherapy has not been shown to have clear benefit for survival. Larger and more studies with other risk stratifications including local factors are needed to improve global and regional-specific practices in the treatment of Dukes B and C colon cancer.

Inconclusion, in a regional context, this study supports current evidence from the West that adjuvant chemotherapy improves survival in Dukes C colon cancer post curative surgery. However, a clear benefit is yet to be proven for Dukes B disease although there was evidence showing the trend supporting the use of chemotherapy in high risks patients and our results suggest survival improvement in selected cases.

\section{References}

Andre T, Boni C, Mounedji-Boudiaf L, et al (2004). Oxaliplatin, fluorouracil, and leucovorin as adjuvant treatment for colon cancer. N Engl J Med, 350, 2343-51.

Andre T, Boni C, Navarro M, et al (2009). Improved overall survival with oxaliplatin, fluorouracil, and leucovorin as adjuvant treatment in stage II or III colon cancer in the MOSAIC trial. J Clin Oncol, 27, 3109-16.

Ariffin OZ, Nor Saleha (2011). Malaysia Cancer Statistics-Data and Figures. National Cancer Registry Report 2007.

Benson AB, 3rd, Schrag D, Somerfield MR, et al (2004). American Society of Clinical Oncology recommendations on adjuvant chemotherapy for stage II colon cancer. J Clin Oncol, 22, 3408-19.

Bozkurt O, Inanc M, Turkmen E, et al (2014). Clinicopathological characteristics and prognosis of patients according to recurrence time after curative resection for colorectal cancer. Asian Pac J Cancer Prev, 15, 9277-81.

Buyse M, Piedbois P (2001). Should Dukes' B patients receive adjuvant therapy? A statistical perspective. Semin Oncol, 28, 20-4.

Chang GJ, Rodriguez-Bigas MA, Skibber JM, et al (2007). Lymph node evaluation and survival after curative resection of colon cancer: systematic review. J Natl Cancer Inst, 99 , 433-41.

Chen SL, Bilchik AJ (2006). More extensive nodal dissection improves survival for stages I to III of colon cancer: a population-based study. Ann Surg, 244, 602-10.

Chiu J, Tang V, Leung R, et al (2014). Efficacy and tolerability of adjuvant oral capecitabine plus intravenous oxaliplatin (XELOX) in Asian patients with colorectal cancer: 4-year analysis. Asian Pac J Cancer Prev, 14, 6585-90.

Chong VH, Abdullah MS, Telisinghe PU, et al (2009). Colorectal cancer: incidence and trend in Brunei Darussalam. Singapore Med J, 50, 1085-9.

Compton CC, Fielding LP, Burgart LJ, et al (2000). Prognostic factors in colorectal cancer. College of american pathologists consensus statement 1999. Arch Pathol Lab Med, 124, 979-94.

Edge SB, Compton CC (2010). The American Joint Committee on Cancer: the 7th edition of the AJCC cancer staging manual and the future of TNM. Ann Surg Oncol, 17, 1471-4.

Elsamany SA, Alzahrani AS, Mohamed MM, et al (2014). Clinico-pathological patterns and survival outcome of colorectal cancer in young patients: western Saudi Arabia experience. Asian Pac J Cancer Prev, 15, 5239-43.

Fang XY, Xu WD, Huang Q, et al (2014). 5,10-Methylenetetrahydrofolate reductase polymorphisms and colon cancer risk: a meta-analysis. Asian Pac J Cancer Prev, 15, 8245-50.

Galizia G, Orditura M, Ferraraccio F, et al (2009). The lymph node ratio is a powerful prognostic factor of node-positive colon cancers undergoing potentially curative surgery. World J Surg, 33, 2704-13.

Kim MJ, Jeong SY, Choi SJ, et al (2015). Survival Paradox Between Stage IIB/C (T4N0) and Stage IIIA(T1-2N1) Colon Cancer. Ann Surg Oncol, 22, 505-12.

Kong CK, Roslani AC, Law CW, et al (2010). Impact of socioeconomic class on colorectal cancer patient outcomes in Kuala Lumpur and Kuching, Malaysia. Asian Pac J Cancer Prev, 11, 969-74.

Magaji BA, Moy FM, Roslani AC, et al (2014). Descriptive epidemiology of colorectal cancer in University Malaya Medical Centre, 2001 to 2010. Asian Pac J Cancer Prev, 15, 6059-64.

McKenzie S, Nelson R, Mailey B, et al (2011). Adjuvant 

chemotherapy improves survival in patients with American Joint Committee on Cancer stage II colon cancer. Cancer, 117, 5493-9.

Morris EJ, Maughan NJ, Forman D, et al (2007). Who to treat with adjuvant therapy in Dukes B/stage II colorectal cancer? The need for high quality pathology. Gut, 56, 1419-25.

Morrison DS, Parr CL, Lam TH, et al (2013). Behavioural and metabolic risk factors for mortality from colon and rectum cancer: analysis of data from the Asia-Pacific Cohort Studies Collaboration. Asian Pac J Cancer Prev, 14, 1083-7.

Nadoshan JJ, Omranipour R, Beiki O, et al (2013). Prognostic value of lymph node ratios in node positive rectal cancer treated with preoperative chemoradiation. Asian Pac J Cancer Prev, 14, 3769-72.

O'Connell JB, Maggard MA, Liu JH, et al (2004). Do young colon cancer patients have worse outcomes? World J Surg, 28, 558-62.

O'Connor ES, Greenblatt DY, LoConte NK, et al (2011). Adjuvant chemotherapy for stage II colon cancer with poor prognostic features. J Clin Oncol, 29, 3381-8.

Omranipour R, Mahmoodzadeh H, Safavi F (2014). Prevalence of local recurrence of colorectal cancer at the Iranian Cancer Institute. Asian Pac J Cancer Prev, 15, 8587-9.

Pourhoseingholi MA (2012). Increased burden of colorectal cancer in Asia. World J Gastrointest Oncol, 4, 68-70.

Quah HM, Chou JF, Gonen M, et al (2008). Identification of patients with high-risk stage II colon cancer for adjuvant therapy. Dis Colon Rectum, 51, 503-7.

Quasar Collaborative G, Gray R, Barnwell J, et al (2007). Adjuvant chemotherapy versus observation in patients with colorectal cancer: a randomised study. Lancet, 370, 2020-9.

Rosenberg R, Friederichs J, Schuster T, et al (2008). Prognosis of patients with colorectal cancer is associated with lymph node ratio: a single-center analysis of 3,026 patients over a 25-year time period. Ann Surg, 248, 968-78.

Schrag D, Rifas-Shiman S, Saltz L, et al (2002). Adjuvant chemotherapy use for Medicare beneficiaries with stage II colon cancer. J Clin Oncol, 20, 3999-4005.

Shah SA, Neoh HM, Rahim SS, et al (2014). Spatial analysis of colorectal cancer cases in Kuala Lumpur. Asian Pac J Cancer Prev, 15, 1149-54.

Shepherd NA, Baxter KJ, Love SB (1997). The prognostic importance of peritoneal involvement in colonic cancer: a prospective evaluation. Gastroenterology, 112, 1096-102.

Tsukuma H, Ioka A, Tanaka M (2011). [Incidence and mortality of colorectal cancer--international comparison]. Nihon Rinsho, 69, 45-50.

Vather R, Sammour T, Kahokehr A, et al (2009). Lymph node evaluation and long-term survival in Stage II and Stage III colon cancer: a national study. Ann Surg Oncol, 16, 585-93.

Wilkinson NW, Yothers G, Lopa S, et al (2010). Long-term survival results of surgery alone versus surgery plus 5-fluorouracil and leucovorin for stage II and stage III colon cancer: pooled analysis of NSABP C-01 through C-05. A baseline from which to compare modern adjuvant trials. Ann Surg Oncol, 17, 959-66.

Wong AC, Stock S, Schrag D, et al (2014). Physicians' beliefs about the benefits and risks of adjuvant therapies for stage ii and stage iii colorectal cancer. J Oncol Pract, 10, 360-7.

Yahaya MA, Chin PY, Sulaiman LH, et al (2011). Annual report Ministry of Health, Malaysia.

Yee YK, Tan VP, Chan P, et al (2009). Epidemiology of colorectal cancer in Asia. J Gastroenterol Hepatol, 24, 1810-6.

Yun JA, Kim HC, Kim SH, et al (2014). Prognostic significance of perineural invasion in stage IIA colon cancer. ANZ J Surg. 